\title{
Análise das infecções de sítio cirúrgico em pacientes ortopédicos de um hospital do
}

\section{Paraná}

\author{
Analysis of surgical site infections in orthopedic patients in a hospital in Paraná \\ Análisis de las infecciones del sitio quirúrgico en pacientes ortopédicos en un hospital de Paraná
}

Recebido: 17/12/2021 | Revisado: 24/12/2021 | Aceito: 27/12/2021 | Publicado: 27/12/2021

Laura Bazzi Longo

ORCID: https://orcid.org/0000-0003-1396-1524

Universidade Estadual de Ponta Grossa, Brasil E-mail: laurabazzilongo@hotmail.com

Amanda de Souza Lemos

ORCID: https://orcid.org/0000-0002-8837-2823

Universidade Estadual de Ponta Grossa, Brasil E-mail: amanda soule@hotmail.com José Carlos Rebuglio Vellosa

ORCID: https://orcid.org/0000-0003-4747-9197

Universidade Estadual de Ponta Grossa, Brasil E-mail: vellosajcr@hotmail.com

Elisangela Gueiber Montes

ORCID: https://orcid.org/0000-0003-1694-085X

Universidade Estadual de Ponta Grossa, Brasil E-mail: elisangela.gueiber@uol.com.br

\begin{abstract}
Resumo
As infecções de sítio cirúrgico são encontradas em 14-16\% dos pacientes hospitalizados, sendo que, as relacionadas a cirurgias ortopédicas são consideradas severas. Portanto, a determinação de fatores de risco, é necessária, pois permite identificar situações que predisponham o desenvolvimento dessa infecção. Com o objetivo de elencar os fatores de riscos relacionados as infecções de sítio cirúrgico em um hospital universitário do Paraná, em um estudo transversal descritivo retrospectivo, foram analisados 125 prontuários de indivíduos submetidos a cirurgias ortopédicas e que desenvolveram a infecção no pós-operatório. Assim, foi possível encontrar um perfil de pacientes mais susceptível a desenvolver essa complicação, na qual, $41,0 \%$ dos pacientes foram identificados como saudáveis no pré-operatório, 61,6\% das cirurgias tinham um caráter limpo, 63,2\% utilizaram implante ortopédico no seu tratamento e o tratamento cirúrgico de fraturas fechadas prevaleceu. A incidência global da infecção foi de 2,0\% e os micro-organismos mais prevalentes na amostra foram o Staphylococcus aureus, Enterobacter cloacae e Escherichia coli com 7,2\% cada. Comparando-se com a literatura abordada, diferenças são encontradas na prevalência dos fatores de risco, devido às diferenças de cada população. A identificação desses fatores de risco é de suma importância para a prevenção das infecções de sítio cirúrgico. Por fim, novos estudos ainda devem ser realizados com o objetivo de validar os fatores de risco encontrados.
\end{abstract}

Palavras-chave: Infecção de sítio cirúrgico; Cirurgias ortopédicas; Fatores de risco.

\begin{abstract}
Surgical site infections are found in $14-16 \%$ of hospitalized patients, and those related to orthopedic surgeries are considered severe. Therefore, the determination of risk factors is necessary to identify situations that predispose to the development of this infection. Aiming to list the risk factors related to surgical site infections at a university hospital in Paraná, in a retrospective cross-sectional descriptive study, 125 medical records of individuals who had undergone orthopedic surgery and developed postoperative infection were analyzed. Thus, it was possible to find a profile of patients more susceptible to developing this complication, in which, $41.0 \%$ of the patients were identified as healthy preoperatively, $61.6 \%$ of the surgeries were clean, $63.2 \%$ used orthopedic implant in their treatment and surgical treatment of closed fractures prevailed. The overall incidence of infection was $2.0 \%$ and the most prevalent microorganisms in the sample were Staphylococcus aureus, Enterobacter cloacae and Escherichia coli with 7.2\% each. Comparing with the literature covered, differences are found in the prevalence of risk factors, due to the differences of each population. The identification of these risk factors is of utmost importance for the prevention of surgical site infections. Finally, new studies should still be carried out to validate the risk factors found.
\end{abstract}

Keywords: Surgical site infection; Orthopedic surgery; Risk factors. 


\begin{abstract}
Resumen
Las infecciones del sitio quirúrgico se encuentran en el 14-16\% de los pacientes hospitalizados, y las relacionadas con las cirugías ortopédicas se consideran graves. Por lo tanto, la determinación de los factores de riesgo es necesaria para identificar las situaciones que predisponen al desarrollo de esta infección. Con el objetivo de enumerar los factores de riesgo relacionados con las infecciones del sitio quirúrgico en un hospital universitario de Paraná, en un estudio descriptivo transversal retrospectivo, se analizaron 125 historias clínicas de personas que se sometieron a cirugías ortopédicas y desarrollaron una infección postoperatoria. Así, se pudo encontrar un perfil de pacientes más susceptibles de desarrollar esta complicación, en el cual, el 41,0\% de los pacientes fueron identificados como sanos en el preoperatorio, el 61,6\% de las cirugías fueron limpias, el 63,2\% utilizaron implantes ortopédicos en su tratamiento y predominó el tratamiento quirúrgico de las fracturas cerradas. La incidencia global de la infección fue del 2,0\% y los microorganismos más prevalentes en la muestra fueron Staphylococcus aureus, Enterobacter cloacae y Escherichia coli, con un 7,2\% cada uno. Comparando con la literatura abordada, se encuentran diferencias en la prevalencia de los factores de riesgo, debido a las diferencias de cada población. La identificación de estos factores de riesgo es de suma importancia para la prevención de las infecciones del sitio quirúrgico. Por último, deberían realizarse más estudios para validar los factores de riesgo encontrados.
\end{abstract}

Palabras clave: Infección del sitio quirúrgico; Cirugías ortopédicas; Factores de riesgo.

\title{
1. Introdução
}

A Infecção Relacionada à Assistência à Saúde (IRAS) é definida como qualquer infecção que pode ser adquirida nos estabelecimentos de saúde e está relacionada à assistência prestada. Atualmente, esse tipo de infecção é considerado um problema de saúde pública e representa uma das complicações que mais acometem os pacientes internados (Engelman, 2016). Dentre elas, destaca-se a Infecção de Sítio Cirúrgico (ISC), que pode ocorrer na incisão cirúrgica ou em tecidos manipulados durante a operação, classificada de acordo com sua topografia em incisional superficial, profunda e, de órgão/cavidade (Mangram et al., 1999).

Em relação a sua classificação topográfica, segundo o Centers For Disease Control and Prevention, (2017), a ISC incisional superficial envolve apenas incisões de pele e tecido subcutâneo e, o seu aparecimento ocorre nos primeiros 30 dias após a cirurgia. A profunda, acomete incisões dos tecidos moles profundos (fáscia e músculo) e sua vigilância ocorre entre os primeiros 30 até os 90 dias depois da cirurgia, dependendo do uso ou não de implantes durante o ato operatório. Já a de órgão/cavidade, envolve qualquer parte do corpo mais profunda que é aberta ou manipulada durante o procedimento cirúrgico e o seu desenvolvimento ocorre entre os primeiros 30 até 90 dias pós-operatório, dependendo do uso ou não de implantes (ANVISA, 2017).

A ISC, nos dias de hoje, ainda é considerada um dos principais riscos à segurança dos pacientes nos serviços de saúde do Brasil, onde a sua incidência ocupa o 3o lugar entre as IRAS, sendo encontrada em 14-16\% dos pacientes hospitalizados, segundo estudos brasileiros. Acredita-se que, aproximadamente, 60\% dos casos poderiam ser evitados através da aplicação de medidas de orientação e prevenção recomendadas em diretrizes e manuais. De acordo com a Sociedade Americana de Epidemiologia Hospitalar (SHEA) e com a Sociedade Americana de Doenças Infecciosas (IDSA), em 2014, nos Estados Unidos da América (EUA), a ISC acometeu 2-5\% dos pacientes que foram submetidos à procedimentos cirúrgicos, totalizando 160.000 a 300.000 episódios de ISC por ano no país (ANVISA, 2017).

Apesar dos avanços significativos nas técnicas cirúrgicas, salas de cirurgias modernas e medidas perioperatórias preventivas, como a profilaxia antimicrobiana intravenosa e a antissepsia cutânea pré-operatória, as infecções de ferida operatória continuam a ser um problema nas cirurgias (Greene, 2012). Além de aumentarem o risco de morbimortalidade do paciente, esse tipo de infecção pode acarretar em graves consequências econômicas ao prolongar a estadia do paciente em média de sete a onze dias, aumentar a chance de readmissão hospitalar, cirurgias adicionais, e, consequentemente, elevar os gastos assistenciais com o tratamento, que podem chegar a US\$1,6 bilhão anuais (Mendes et al., 2020).

Dentre os subtipos dessa infecção, as relacionadas a cirurgias ortopédicas são consideradas preocupantes. Em geral, 
nessas cirurgias, são utilizadas próteses, fato esse, que eleva o risco de infecção, pode levar à amputação do membro operado e até à morte do paciente. Alguns estudos do Brasil apontam que a ocorrência de ISC ortopédica varia de 1,4 a 40,3\% (Ercole, Chianca, Duarte, Starling, \& Carneiro, 2011; Ana Lúcia Lei Munhoz Lima et al., 2004). De acordo com Ercole, Franco, Macieira, Wenceslau, Resende et al. (2011), em um grande hospital público brasileiro, deparou-se com uma incidência desse tipo de complicação pós-operatória de 1,8\%. Ademais, segundo Cunha (2019), em um hospital geral de Belo Horizonte, mas em procedimentos com uso de implantes ortopédicos, a taxa de ISC encontrada foi de 4,03\%.

Em relação aos fatores de risco relacionados à infecção ortopédica, destacam-se as comorbidades prévias do paciente, período de internação antes da cirurgia prolongado, tempo cirúrgico, preparo da pele, técnica de redução dos microrganismos de toda a equipe cirúrgica, situação da sala cirúrgica, quantidade de profissionais dentro da sala, técnica e destreza do cirurgião, utilização de próteses, entre muitas outras variáveis (Ercole, Franco, Macieira, Wenceslau, de Resende, et al., 2011).

As graves consequências causadas aos pacientes que desenvolvem a ISC determinam a necessidade de esforços para a criação de estratégias, a fim de que esse tipo de infecção seja prevenido. De um lado, uma das estratégias utilizadas é a determinação de condições de risco, o que permite identificar situações ou fatores clínicos que predisponham ao desenvolvimento da ISC (Carvalho et al., 2017).

A identificação dos fatores de risco para essa infecção contribui para a adoção precoce de intervenções por parte dos profissionais de saúde, objetivando minimizar esse tipo de complicação pós-operatória (Carvalho et al., 2017). Dessa maneira, nos últimos anos, diversos estudos acerca das condições de risco relacionados a ela foram feitos, no entanto, devido à característica de cada população, percebe-se diferença nas taxas de infecção e nos fatores de risco. Portanto, é de extrema importância que estudos multicêntricos sejam realizados, a fim de conhecer a gravidade desta infecção (Franco et al., 2015).

Do outro lado, o uso de antimicrobianos faz parte do conjunto de medidas utilizadas tanto com a finalidade de diminuir as infecções de ferida operatória, através da profilaxia antibiótica, como também do seu tratamento, levando em conta as recomendações gerais e especificas para cada tipo de cirurgia (Gebrim et al., 2014). Assim, a Organização Mundial da Saúde (OMS) e outras sociedades emitiram diretrizes para prevenir esse tipo de infecção, mas sua implementação continua sendo um desafio (Fontes, 2010; Roth et al., 2019).

Quando a infecção já está estabelecida, o rápido diagnóstico clínico e laboratorial, associado às condutas terapêuticas adequadas, contribuem para aumentar a possibilidade de resolução definitiva da infecção. $\mathrm{O}$ uso irracional dessa terapêutica, na prática médica, é um fato conhecido e que pode acarretar riscos à segurança dos pacientes (Ana Lucia Lei Munhoz Lima \& de Oliveira, 2010).

Dessa forma, esse estudo tem como objetivo analisar os fatores de riscos relacionados à pacientes submetidos à cirurgia ortopédica, e que, posteriormente, desenvolveram ISC em um hospital universitário do Paraná, bem como a conduta antibiótica escolhida em cada caso. A relevância desse estudo está pautada no âmbito de contribuir com a melhoria das estratégias de vigilância para essa complicação ortopédicas do nosocômio de estudo já existentes, com o objetivo de prevenir esse tipo de infecção.

\section{Metodologia}

Trata-se de um estudo descritivo transversal retrospectivo, como descreve Hochman et al. (2005) em seu trabalho, no qual foram selecionados e analisados os prontuários eletrônicos de pacientes submetidos a cirurgias ortopédicas e que, posteriormente, desenvolveram ISC no Hospital Universitário Regional Wallace Thadeu de Mello e Silva, do Paraná, no período de janeiro de 2017 a dezembro de 2020.

Todos os prontuários revisados estavam cadastrados no banco de dados do Núcleo de Epidemiologia e Controle de 
Infecção Hospitalar (NUCIH) do Hospital Universitário e foram acessados através da plataforma de registro de prontuários eletrônicos no Sistema de Gestão Hospitalar e Ambulatorial do Sistema Único de Saúde (GSUS).

Foram incluídos nesse estudo, os dados dos pacientes que realizaram qualquer tipo de cirurgia ortopédica no referido nosocômio e que, posteriormente, manifestaram sinais de infecção no sítio da cirurgia no período de 30 dias, nas cirurgias sem a utilização de próteses ortopédicas, ou que manifestaram esses sinais dentro de 90 dias, nos casos em que houve a utilização de implantes ortopédicos.

Os critérios utilizados para caracterizar quais seriam esses sinais, foram baseados na referência bibliográfica utilizada pelo hospital de estudo: o Caderno de Critérios Diagnósticos de Infecções Relacionadas à Assistência à Saúde da ANVISA (2017). Como no prontuário eletrônico não continha a classificação da ISC (superficial, profunda ou de órgão/cavidade), qualquer critério de ISC superficial encontrado em menos de 30 dias após o procedimento, em cirurgias que não utilizaram implante ortopédico, ou qualquer critério de ISC profunda ou de órgão/cavidade encontrado em até 30 dias de pós-operatório (em cirurgias que não fizeram uso de implantes) ou em até 90 dias de pós-operatório (em cirurgias que utilizaram implantes), foram considerados para a inclusão do caso e para a contagem dos dias de evolução para ISC.

Assim, inicialmente foram relatados 131 casos de infecção de 2017-2020 cadastrados no NUCIH, sendo que desses, 6 foram excluídos por desenvolverem a infecção depois de 30 dias, em cirurgias sem o uso de implantes, ou depois 90 dias com o uso de implantes, restando 125 casos para análise, dos quais 27 eram referentes à indivíduos que sofreram mais de um caso de infecção no período estudado, pelo mesmo procedimento ou, por um procedimento diferente do anterior. Para facilitar a análise dos dados e evitar vieses, o número da amostra considerada foi de 125 para a maioria das variáveis.

Os pacientes que realizaram a cirurgia de caráter eletivo, tiveram seu risco cirúrgico classificado através do ASA (American Society of Anesthesiology), que conforme as comorbidades do paciente, o classifica de I-VI, sendo I o menor risco cirúrgico e VI, o maior. Conforme o protocolo da instituição, apenas cirurgias de caráter eletivo necessitam dessa avaliação no pré-operatório, sendo dispensada em casos de emergência. Além disso, devido a pandemia do COVID-19 que se iniciou em 2020 aqui no Brasil, os pacientes submetidos a cirurgias neste ano não tiveram seu risco cirúrgico avaliado, pois passou a ser dispensável. Dessa forma, para não prejudicar os resultados do trabalho e utilizando os critérios mais atualizados preconizados pela Sociedade Americana de Anestesiologistas para a avaliação do ASA, todos os pacientes realizaram cirurgias não emergenciais foram reclassificados ou classificados durante a coleta de dados, conforme a Tabela 1. Ademais, para melhor análise da variável, o ASA foi dicotomizado em 2 grupos: ASA I e ASA II-III.

Tabela 1 - Atuais classificações e definições do ASA (2020).

\begin{tabular}{c|l}
\hline Classificação do ASA & \multicolumn{1}{c}{ Definição } \\
\hline ASA I & Paciente normal e saudável \\
\hline ASA II & Paciente com doença sistêmica leve \\
\hline ASA III & Paciente com doença sistêmica grave \\
\hline ASA IV & $\begin{array}{l}\text { Paciente com doença sistêmica grave que é uma } \\
\text { constante ameaça à vida }\end{array}$ \\
\hline ASA V & $\begin{array}{l}\text { Paciente moribundo que não se espera que sobreviva } \\
\text { sem a operação }\end{array}$ \\
\hline ASA VI & Paciente com morte cerebral declarada \\
\hline
\end{tabular}

Adaptado de: ASA Physical Status Classification System, Committee of Oversight: Economics (Approved by the ASA House of Delegates on October 15, 2014, and last amended on December 13, 2020).

Para melhor visualização do tempo de internação hospitalar pré-operatória, essa variável foi dividida em cirurgias que precisaram de menos de 4 dias de hospitalização antes da operação e cirurgias que precisaram de mais de 4 dias. Ainda, para fins 
estatísticos, como o tempo mínimo de permanência pré-operatório foi de menos de 24 horas, esse tempo em dia foi considerado igual a zero.

As variáveis coletadas para análise foram relacionadas à cirurgia como, tipo de procedimento, tempo internação antes da cirurgia, uso de anestesia geral, uso de prótese ortopédica, caráter eletivo ou não do procedimento e potencial de contaminação da cirurgia, como também, variáveis relacionadas ao paciente como, gênero, idade e classificação do ASA e, relacionadas à infecção, como período em que a infecção se desenvolveu (alta/internado), número de óbitos, tempo para evolução para ISC, necessidade de novo atendimento pelo mesmo caso de ISC e agente etiológico encontrado. Da mesma forma, foram analisadas variáveis relacionadas a conduta antimicrobiana escolhida, como o uso de antibioticoprofilaxia, uso de terapia antibiótica empírica e esquema terapêutico escolhido.

Em um segundo momento, foi utilizado o programa Microsoft Office Excel®, para a construção de gráficos e tabelas. Além disso, os dados coletados passaram por uma análise estatística descritiva, por esse mesmo programa, onde foram realizadas medidas de frequência absoluta e relativa, medidas de tendência central e de variabilidade.

O estudo foi realizado após a autorização do Comitê de Ética em Pesquisa com o parecer de no 4.110 .874 , e da Diretoria Acadêmica e o Centro de Estudos, Pesquisa e Desenvolvimento Humano do Hospital Universitário Regional dos Campos Gerais Wallace Thadeu de Mello e Silva, de $n^{\circ} 8$.

\section{Resultados e Discussão}

Durante o período analisado, foram realizadas 6.123 cirurgias ortopédicas no nosocômio de estudo, indicando uma incidência global da ISC de 2,0\%. Essa taxa obtida se encontra abaixo dos valores encontrados em grande parte da literatura, que tiveram suas taxas variando de 3,4\% até 10,5\% (Carvalho et al., 2017; Medina-Cuadros et al., 1996; Onyekwelu et al., 2017). Em contrapartida, Ercole, Franco, Macieira, Wenceslau, Resende et al. (2011), encontrou uma taxa de ocorrência de 1,8\% para o seu período de estudo, sendo também, inferior aos parâmetros encontrados na literatura e, inclusive inferior aos nossos resultados.

Foram avaliados 98 prontuários eletrônicos de pacientes que realizaram 107 cirurgia ortopédicas e que causaram 125 casos de ISC. A média da idade desses indivíduos foi de 47,5 anos (desvio padrão: 19,2) e a mediana foi de 49 anos. Verificouse também, que a idade mínima dos pacientes submetidos a operações ortopédicas foi de 9 anos e a máxima de 89 anos.

Nesse estudo, encontrou-se uma prevalência do gênero masculino (68,0\%), podendo também ser visualizada em grande parte dos estudos que analisaram tal variável (Ercole, Franco, Macieira, Wenceslau, Resende, et al., 2011; Ren et al., 2019; Onyekwelu et al., 2017; Ortega et al., 2012; Moon et al., 2019). Por outro lado, segundo os resultados de Carvalho et al. (2017), dentre os pacientes ortopédicos que desenvolveram ISC, a maioria era do gênero feminino.

Com relação ao tempo de permanência pré-operatória, a média foi de 4,0 dias (desvio padrão: 7,0), com uma mediana de 1 dia. Além disso, evidenciou-se que o tempo mínimo de permanência pré-operatório foi de menos de 24 horas, sendo que, isso significa que estes pacientes realizaram a cirurgia no mesmo dia em que foram internados. Já o tempo máximo de permanência antes do procedimento foi de 42 dias.

Dessa forma, nesse estudo, 72,0\% das cirurgias aconteceram com um tempo de permanência pré-operatória menor ou igual a 4 dias. No entanto, é sabido que um tempo prolongado de internação, antes da realização da cirurgia, têm sido associados com o aumento do risco de ISC. Isso pode ser explicado pelo fato de que um tempo prolongado de permanência no hospital, antes da cirurgia, pode provocar modificações na microbiota do paciente e consequente colonização por micro-organismos presentes no ambiente hospitalar (Mangram et al., 1999). Todavia, os dados da literatura apoiaram nossos resultados, nos quais, 74,6\% dos pacientes que evoluíram com ISC permaneceram 4 dias ou menos internados no pré-operatório (Ercole, Franco, 
Macieira, Wenceslau, Resende, et al., 2011). Outro caso, refere-se a uma coorte não concorrente de 149 pacientes que realizaram artroplastia de quadril e joelho, em um hospital de Belo Horizonte, no qual, 6 casos de ISC foram notificados. Desses 6 pacientes, $5(83,3 \%)$ apresentaram tempo de internação pré-cirúrgico menor ou igual a 1 dia de internação (Cunha, 2019).

Dos 100 casos que receberam a nota do ASA, 59,0\% possuíam ASA II ou III, ou seja, possuíam um risco cirúrgico de mortalidade perioperatória maior que os pacientes categorizados como ASA I. Dessa forma, 41,0\% foram classificados como saudáveis (ASA I). Na literatura, o valor encontrado foi ainda inferior, no qual, 17,5\% dos pacientes que evoluíram com ISC, foram classificados com ASA I (Ercole, Franco, Macieira, Wenceslau, Resende, et al., 2011). Em contrapartida, segundo Ribeiro et al. (2013), dos 16 pacientes com ISC, 14 foram classificados como saudáveis ou com leve comprometimento, ou seja, ASA I ou II respectivamente. Os ASA IV, V e VI não foram encontrados em nenhum paciente da nossa amostra.

Por outro lado, em relação ao potencial de contaminação cirúrgica, 61,6\% (77) delas eram limpas, ou seja, segundo o presente estudo, nota-se que o grau de contaminação da cirurgia não influenciou no risco de ISC. De acordo com os riscos de contaminação, as cirurgias são classificadas em: limpas, potencialmente contaminadas, contaminadas e infectadas. Essa classificação é feita por um profissional da saúde, seguindo os critérios do hospital em questão, momentos antes da cirurgia. Esse fato é corroborado por Ercole, Franco, Macieira, Wenceslau, Resende et al. (2011), no qual 82\% dos pacientes que evoluíram com ISC tiveram o potencial de contaminação da ferida cirúrgica classificado como limpa. No entanto, outros estudos não tiveram o mesmo desfecho e as feridas cirúrgicas potencialmente contaminadas, contaminadas e infectadas prevaleceram nos pacientes que desenvolveram ISC (Onyekwelu et al., 2017; Carvalho et al., 2017). A distribuição do potencial de contaminação cirúrgica referente a todos os pacientes analisados está reunida no Gráfico 1.

Gráfico 1 - Distribuição do potencial de contaminação das cirurgias ortopédicas que evoluíram com ISC, entre 2017 e 2020.

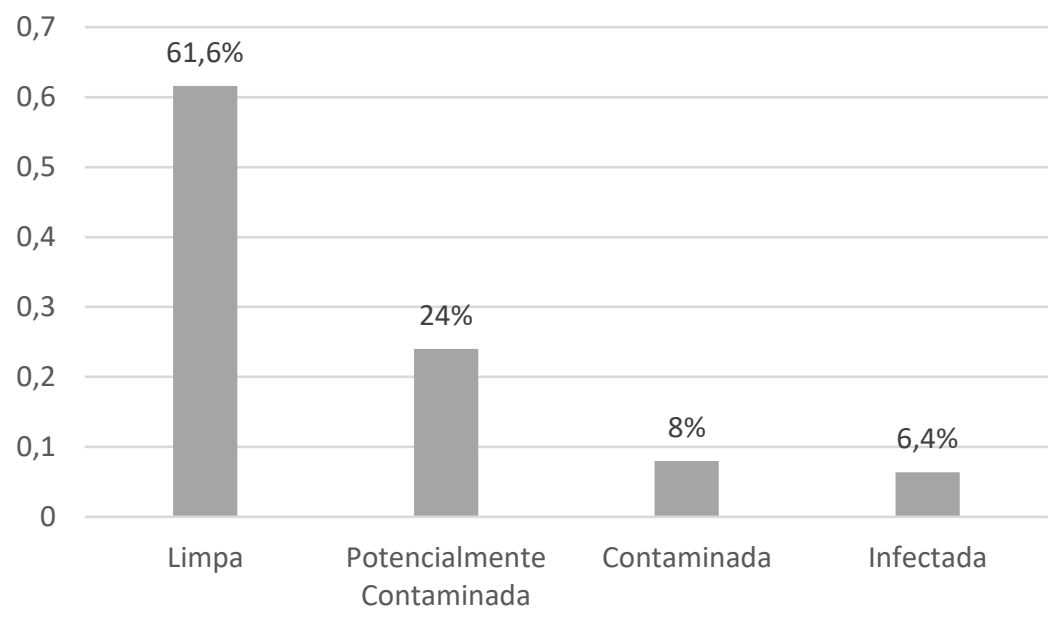

Fonte: Autores.

O não uso de anestesia geral prevaleceu nesse estudo, totalizando 68,8\% das cirurgias ortopédicas que evoluíram para ISC. Na literatura, os dados são conflitantes, em uma coorte histórica realizada em um hospital público de Minas Gerais, no Brasil, 52,3\% dos casos de ISC não tiveram anestesia geral aplicada no ato operatório (Ercole, Franco, Macieira, Wenceslau, Resende, et al., 2011). Em contrapartida, em um estudo de coorte, não concorrente, realizado em um hospital de grande porte de Belo Horizonte, no Brasil, a maioria dos casos de infecção de ferida cirúrgica utilizaram anestesia geral no ato operatório (Carvalho et al., 2017).

Em relação ao caráter da cirurgia, ela pode ser eletiva, com agendamento prévio e com preparação do paciente e da 
equipe, ou, de emergência. Na análise do presente estudo, o caráter eletivo das cirurgias foi prevalente, totalizando $80,0 \%$ dos casos, sugerindo que a situação de emergência da cirurgia ortopédica, não tem relação com um maior risco de evolução para ISC. Isso é reafirmado na literatura, na qual, segundo Ercole, Franco, Macieira, Wenceslau, Resende et al. (2011), 96,8\% dos pacientes com ISC, submeteram-se a cirurgias eletivas. No entanto, é interessante ressaltar que em um estudo chinês que analisou ISC apenas na população idosa (65 anos ou mais), $84,1 \%$ dos idosos, tiveram a infecção relacionada a cirurgias de caráter emergencial. Isso pode ser explicado, pelo fato de que, estudos mostraram uma maior prevalência de ISC em cirurgias de pacientes geriátricos (Le et al., 2020).

Além disso, verificou-se também que $63,2 \%$ das cirurgias ortopédicas que, posteriormente culminaram com ISC, utilizaram implante ortopédico no seu tratamento. Esse dado foi muito parecido com o encontrado na literatura onde 56\% dos pacientes que culminaram com ISC, utilizaram implantes ortopédicos no ato operatório (Ercole, Franco, Macieira, Wenceslau, Resende, et al., 2011).

Sobre os tipos de procedimentos mais frequentes dentre as cirurgias analisadas, que evoluíram com ISC, em ordem decrescente foram: tratamento cirúrgico de fratura fechada (TCFF) em 25,6\%; tratamento cirúrgico de fratura exposta (TCFE) em 20,0\%; artroplastia de quadril (ARTQ) em 9,6\%; amputação (AMP) em 8,8\%; tratamento cirúrgico de fusão/artrodese (F/A) em 6,4\%; reparo de tendões/ligamentos (REPT/L) em 5,6\%; e artroplastia de joelho (ARTJ) em 3,2\%. Foram agrupadas em “outras cirurgias (OUT)": fasciotomia, retirada de fixador externo meniscectomia, revisão de artroplastia de quadril, retirada de corpo estranho, revisão de artroplastia de joelho, retirada de placas e parafusos, tratamento cirúrgico de fratura não exposta + fasciotomia, tratamento cirúrgico de pseudoartrose, osteostomia, tratamento cirúrgico de fusão/artrodese + reparo de ligamento/tendões, tratamento cirúrgico da doença de Dupuytren, retirada de prótese + tratamento cirúrgico de fusão/artrodese e retirada de prótese de joelho. Esse último grupo correspondeu a 20,8\% das cirurgias analisadas. A frequência de cada procedimento pode ser visualizada na Tabela 2.

Tabela 2 - Frequência dos tipos de procedimentos ortopédicos realizados que evoluíram com ISC, no período de 2017-2020.

\begin{tabular}{|c|c|}
\hline Tipo de procedimento & n (\%) \\
\hline TCFE & $25(20,0)$ \\
\hline TCFF & $32(25,6)$ \\
\hline AMP & $11(8,8)$ \\
\hline F/A & $8(6,4)$ \\
\hline ARTQ & $12(9,6)$ \\
\hline ARTJ & $4(3,2)$ \\
\hline REPT/L & $7(5,6)$ \\
\hline OUT & $26(20,8)$ \\
\hline
\end{tabular}

$\mathrm{n}=$ tamanho da amostra resultante da variável analisada. $(\%)=$ frequência da amostra resultante da variável analisada. TCFE $=$ Tratamento cirúrgico de fratura exposta. TCFF $=$ Tratamento cirúrgico de fratura fechada. AMP= Amputação. F/A: Tratamento cirúrgico de fusão/artrodese. ARTQ= Artroplastia de quadril. REPT/L= Reparo de tendões/ligamentos. ARTJ= Artroplastia de joelho. OUT= outras cirurgias. Sendo elas: fasciotomia, retirada de fixador externo meniscectomia, revisão de artroplastia de quadril, retirada de corpo estranho, revisão de artroplastia de joelho, retirada de placas e parafusos, tratamento cirúrgico de fratura não exposta + fasciotomia, tratamento cirúrgico de pseudoartrose, osteostomia, tratamento cirúrgico de fusão/artrodese + reparo de ligamento/tendões, tratamento cirúrgico da doença de Dupuytren, retirada de prótese + tratamento cirúrgico de fusão/artrodese e Retirada de prótese de joelho. Fonte: Autores. 
Como visto na amostra, o tratamento cirúrgico de fraturas fechadas prevaleceu, totalizando 25,6\% dos casos. Esses resultados foram divergentes na literatura. Em um estudo coreano, a fratura fechada prevaleceu em $65,7 \%$ dos pacientes do grupo com ISC (Moon et al., 2019). Por outro lado, em um estudo realizado no Brasil, com pacientes que passaram por procedimento cirúrgico para fixação de fraturas fechadas ou expostas, a incidência de ISC foi quase quatro vezes maior no grupo de pacientes com fraturas expostas do que naqueles com fraturas fechadas (P. R. Oliveira et al., 2016).

Dentre as ISC, 67,2\% ocorreram durante no período em que o paciente estava de alta, ou seja, os sinais e sintomas relacionados a ISC apareceram fora do ambiente hospitalar e após 72h da alta hospitalar. As infecções cirúrgicas com frequência se manifestam no pós-alta hospitalar, por isso, os pacientes devem ser acompanhados a longo prazo, quando já estão em seu domicílio. Na literatura isso é reafirmado. Em um estudo realizado no Brasil por A. C. Oliveira \& Carvalho (2004), 73,7\% das ISC foram diagnosticadas após a alta. Além disso, de acordo com Ferraz et al. (1995), aproximadamente 95\% das ISC se manifestam em torno do vigésimo primeiro dia após a cirurgia.

Neste estudo, foram encontrados 5 óbitos $(4,0 \%)$ relacionados ao processo de ISC. De acordo com a literatura, em um trabalho realizado na cidade de Durham, nos Estados Unidos, a taxa de letalidade atribuída a infecção foi de 4,3\%. Ademais, extrapolando nossa análise, o estudo também encontrou um risco 2 vezes maior de morte durante a hospitalização pós-operatória em pacientes com ISC, do que pacientes que não desenvolveram a infecção (Kirkland et al., 1999). Todas essas informações relacionadas ao paciente, a infecção e a cirurgia ortopédica estão compiladas na Tabela 3.

Tabela 3 - Fatores referentes ao paciente, à infecção e à cirurgia ortopédica encontrados em uma amostra de 125 casos de infecção de sítio cirúrgico, entre 2017-2020.

\begin{tabular}{|c|c|c|}
\hline Fator & & \\
\hline \multirow[t]{2}{*}{ Gênero } & Feminino & $40(32,0)$ \\
\hline & Masculino & $85(68,0)$ \\
\hline \multirow[t]{2}{*}{ PotC } & Limpas & $77(61,6)$ \\
\hline & Outras (PC, $\mathrm{C}, \mathrm{I})$ & $40(38,4)$ \\
\hline \multirow[t]{2}{*}{ An estesia Geral } & Sim & $39(31,2)$ \\
\hline & Não & $86(68,8)$ \\
\hline \multirow[t]{2}{*}{ ASA } & I & $41(41,0)$ \\
\hline & II e III & $59(59,0)$ \\
\hline \multirow[t]{2}{*}{ Carácter da Cirurgia } & Eletiva & $100(80,0)$ \\
\hline & Emergência & $25(20,0)$ \\
\hline \multirow[t]{2}{*}{ Implante Ortopédico } & $\mathrm{Sim}$ & $79(63,2)$ \\
\hline & Não & $46(36,8)$ \\
\hline \multirow[t]{2}{*}{ Permanência pré-operatória } & $=4$ dias & $90(72,0)$ \\
\hline & $>4$ dias & $35(28,0)$ \\
\hline \multirow[t]{2}{*}{ Periodo } & Alta & $84(67,2)$ \\
\hline & Internado & $41(32,8)$ \\
\hline \multirow[t]{2}{*}{ Óbito } & Sim & $5(4,0)$ \\
\hline & Não & $120(96,0)$ \\
\hline
\end{tabular}

$\mathrm{n}=$ tamanho da amostra resultante da variável analisada. $(\%)=$ frequência da amostra resultante da variável analisada. PotC $=$ Potencial de contaminação da ferida cirúrgica. $\mathrm{PC}=$ potencialmente contaminada. $\mathrm{C}=$ contaminada. $\mathrm{I}=$ infectada. ASA= American Society of Anesthesiology. $\leq=$ menor ou igual. $>=$ maior. Fonte: Autores. 
Referente ao tempo de evolução para ISC, foi considerado o número de dias desde o dia da cirurgia até a data em que apareceram os primeiros sinais de infecção de sítio cirúrgico. Dessa forma, a média de dias de evolução para ISC foi de 23,9 dias (desvio padrão: 20,3), com mediana de 18 e, verificou-se um tempo mínimo de 1 dia e um tempo máximo de 90 dias, sendo que, 76,8\% (96) dos pacientes tiveram seu diagnóstico feito até o trigésimo dia de pós-operatório e 24,8\% procuraram novo atendimento por ISC pelo mesmo procedimento.

Em um estudo de 2011 feito em um hospital público do estado de Minas Gerais com tipo de amostra semelhante ao do presente estudo, a média de dias para evolução para a infecção foi de 95,8 dias (Ercole, Franco, Macieira, Wenceslau, Resende, et al., 2011). Essa discrepância de resultados pode ser explicada pelo fato de que, há alguns anos, segundo a ANVISA (Agência Nacional de Vigilância Sanitária), a ISC era considerada como aquela que tivesse manifestado sinais de infecção no sítio da cirurgia até o trigésimo dia após a cirurgia para procedimentos sem implantes ou até um ano na presença de implantes (ANVISA, 2011). No entanto, a partir do ano de 2017, passou a ser considerada ISC, aquela que ocorre nos primeiros 30 dias após a cirurgia ou até 90 dias, se houver colocação de implantes, exceto os casos de identificação de Micobactéria de Crescimento Rápido (MCR), onde deve-se considerar até 24 meses após realização do ato cirúrgico como critério para diagnóstico de ISC (ANVISA, 2017). Em um estudo mais recente de 2019, em um hospital estadual da região norte do Espírito Santo, cerca de 72,7\% das ISC ortopédicas foram notificadas em menos de 15 dias e 9\% foram notificadas de 16 a 30 dias (Nascimento \& Corradi, 2019).

Os micro-organismos mais prevalentes na amostra foram o Staphylococcus aureus, Enterobacter cloacae e Escherichia coli com 7,2\% cada. Este achado é corroborado pela literatura, a qual afirma que o principal agente causador das ISC é o Staphylococcus aureus. Isso pode ser justificado pela suscetibilidade desta bactéria em aderir a estruturas, como os implantes ortopédicos, contribuindo para formação de biofilme, o qual tem capacidade de resistir a antibióticos e aos mecanismos de defesa do hospedeiro (Miranda, 2017).

De acordo Carvalho et al. (2017), além do Staphylococcus aureus, a Escherichia coli também prevaleceu na sua amostra em 15,3\%. Ademais, um estudo realizado na Sérvia, citou entre as bactérias mais frequentemente isoladas na sua amostra, o Enterobacter spp., que também foi prevalente em nossa amostra (Maksimović et al., 2008). Por fim, em uma pesquisa realizada por Miranda (2017), Staphylococcus aureus foi o micro-organismo de maior prevalência (36,1\%), no entanto, em 11,1\% houve o crescimento de Enterobacter cloacae.

Ressalta-se, que dentro dessa amostra, a prevalência de cultura negativa foi de 18,4\%, ausência de cultura foi de 20,8\% e cultura sem resultado foi de $0,8 \%$. O resultado de todas as culturas da amostra, estão agrupados na Tabela 4. 
Tabela 4 - Resultados de cultura encontrados em amostra de pacientes com ISC ortopédica 2017-2020.

\begin{tabular}{l|c}
\hline \multicolumn{1}{c|}{ Micro-organismo } & $\mathbf{n}(\boldsymbol{\%})$ \\
\hline Staphylococcus aureus & $9(7,2)$ \\
\hline Enterobacter cloacae & $9(7,2)$ \\
\hline Escherichia coli & $9(7,2)$ \\
\hline Staphylococcus aureus (MRSA) & $6(4,8)$ \\
\hline Klebsiella aerogenes & $5(4,0)$ \\
\hline Pseudomonas aeruginosa & $5(4,0)$ \\
\hline Klebsiella pneumoniae & $4(3,2)$ \\
\hline Staphylococcus coagulase negativa & $3(2,4)$ \\
\hline Enterococcus faecium & $2(1,6)$ \\
\hline Escherichia coli $($ ESBL) & $2(1,6)$ \\
\hline Klebsiella pneumoniae (KPC) & $2(1,6)$ \\
\hline Proteus mirabilis & $2(1,6)$ \\
\hline Culturas polimicrobianas & $7(5,6)$ \\
\hline Outros & $10(8,0)$ \\
\hline Culturas negativas & $23(18,4)$ \\
\hline Sem resultado & $1(0,8)$ \\
\hline Sem cultura & $26(20,8)$ \\
\hline
\end{tabular}

$\mathrm{n}=$ tamanho da amostra resultante da variável analisada. frequência da amostra resultante da variável analisada. Culturas polimicrobianas: Staphylococcus aureus + Enterobacter cloacae, Staphylococcus coagulase negativa + Enterococcus faecium, Enterococcus faecalis + Staphylococcus aureus (MRSA), Klebsiella pneumoniae + Enterobacter cloacae, Pseudomonas aeruginosa + Proteus mirabilis, Pseudomonas aeruginosa + Enterobacter cloacae e Pseudomonas aeruginosa + Klebsiella pneumoniae (ESBL); Outros: Streptococcus grupo D não Enterococcus, Acinetobacter baumanii, Aerococcus viridans, Burkholderia cepacia complexo, Citrobacter freundii, Pantoea agglomerans, Enterobacter cloacae (ESBL), Klebsiella oxytoca, Klebsiella oxytoca (ESBL), e Klebsiella pneumoniae (ESBL). Fonte: Autores.

Em relação a porcentagem de pacientes que fizeram o uso da profilaxia antibiótica endovenosa, 90,4\% o fizeram, independente se essa foi feita apenas até 24 horas de pós-operatório ou não. Segundo estudos, a Cefazolina é o antibiótico de escolha para a profilaxia desse tipo de infecção. A profilaxia antimicrobiana cirúrgica é necessária, de acordo com as diretrizes e tem como objetivo diminuir a ocorrência de ISC (Gonçalves et al., 2014). No entanto, a eficácia da profilaxia depende de fatores como, o momento de início da profilaxia, a necessidade de repetição da dose no intraoperatório, bem como o tempo de duração da profilaxia, fatores estes, que o presente estudo não abordou. Além disso, a literatura mostra que, quando a antibioticoprofilaxia é instituída incorretamente, sua eficácia pode ser comprometida (Gebrim et al., 2014).

Já em relação a quantidade de pacientes que fizeram o uso da terapia antibiótica empírica, a prevalência foi de $88,8 \%$, ou seja, a maioria dos pacientes tiveram a antiobioticoterapia prescrita antes do resultado da cultura ou da realização desse exame. No caso de ISC, a terapia empírica é indicada devido à gravidade desse tipo de infecção, e é empregada até que fiquem prontos os exames laboratoriais. Como o Stapylococcus spp é o agente etiológico mais comum desde tipo de infecção, a terapia antiestafilocócica é geralmente a escolhida (Gonçalves et al., 2014).

Acerca do tratamento utilizado, independente do uso empírico ou não, 53,6\% fizeram uso da monoterapia antibiótica, $34,4 \%$ fizeram uso da dupla terapia e 4,8\% fizeram uso da tripla terapia. É importante salientar que 7,2\% da amostra não teve registrado no prontuário se foi feito o uso de alguma terapia antibiótica. Na literatura, verificou-se que em Vieira et al. (2021), 66,6\% dos pacientes notificados fizeram uso de antibióticos, portanto, em 33,4\% dos indivíduos não havia registro de uso de antibióticos. Dentre os esquemas terapêuticos utilizados, os mais usados foram: Cefalotina 1g 6/6h por 7 dias endovenoso (EV) em monototerapia e Ciprofloxacino 400mg 12/12h por 7 dias (EV) + Fosfato de clindamicina 600mg 6/6h por 7 dias (EV) em dupla terapia. Pôde ser observado que em tripla terapia, não houve um esquema que se destacasse na amostra. Em pesquisas semelhantes, dados referentes ao esquema terapêutico antimicrobiano utilizado não foram entrados. 


\section{Conclusão}

A partir dos resultados obtidos, pode-se encontrar um perfil de pacientes que realizaram cirurgias ortopédicas, e que, foram mais susceptíveis a desenvolver ISC, no hospital de estudo. Um período curto de internação pré-cirúrgico, um baixo grau de contaminação cirúrgica, o não uso de anestesia geral, o caráter eletivo da cirurgia e o tratamento cirúrgico de fraturas fechadas prevaleceram nos indivíduos com a infecção. Por outro lado, o uso de implantes ortopédicos e um ASA II e III foram predominantes na amostra de pacientes com a infecção. Isso mostra que, com relação literatura abordada, as características de cada população fazem com que encontremos diferenças nos resultados da prevalência de cada fator de risco e por muitas vezes contradizentes com o que se é esperado, quando analisado na prática.

Dessa forma, é de extrema relevância o presente estudo, uma vez que teve como objetivo analisar os fatores de riscos relacionados à pacientes submetidos a cirurgias ortopédicas, e que, no pós-operatório desenvolveram esse tipo de complicação, a fim de conhecer a gravidade das ISC e os variados padrões em que ela pode se apresentar. Ademais, a identificação dessas condições de risco é de suma importância para a prevenção delas.

Em relação à prevenção, o uso da profilaxia antibiótica, apesar de ter sido utilizada na grande maioria da amostra, a maneira como ela foi administrada, não foi analisada pelo estudo, o que pode ter influenciado na prevalência do seu uso em pacientes com a infecção.

Quanto ao conhecimento sobre os microrganismos e sua prevalência, é de suma importância para a equipe hospitalar, permitindo o uso de antimicrobianos mais adequados, bem como evitando seu uso indiscriminado.

Por fim, novos estudos ainda devem ser realizados com o objetivo de validar os fatores de risco encontrados nesta amostra, uma vez que, confirmada a associação, a identificação deles corrobora para a adoção precoce de intervenções a fim de evitar esse tipo de infecção. Além disso, a identificação de condições de risco em infecções de sítio cirúrgico em pacientes de outras especialidades cirúrgicas também deve ser pesquisada, devido a gravidade desse tipo de complicação pós-cirúrgica, que aumenta o risco de morbimortalidade do individuo acometido e acarreta em graves consequências econômicas no sistema de saúde do país.

\section{Referências}

ANVISA. (2011). Cirurgias com implantes/próteses: Critérios Nacionais de Infecções relacionadas a Assistência à Saúde. Agência Nacional de Vigilância Sanitária, 1- 27.

ANVISA. (2017). Critérios Diagnósticos de Infecção Relacionada à Assistência à Saúde. Série Segurança do Paciente e Qualidade em Serviços de Saúde, 189.

Carvalho, R. L. R. de, Campos, C. C., Franco, L. M. de C., Rocha, A. de M., \& Ercole, F. F. (2017). Incidence and risk factors for surgical site infection in general surgeries. Revista Latino-Americana de Enfermagem, 25(2848), 1-8. https://doi.org/10.1590/1518-8345.1502.2848

Cunha, R. C. G. (2019). Incidência e fatores de risco para infecções de sítio cirúrgico ortopédicas com uso de prótese: coorte não concorrente (Tese de Mestrado, Universidade Federal de Minas Gerais, Belo Horizonte). https://repositorio.ufmg.br/handle/1843/30244

Engelman, B. (2016). Fatores de risco para infecção relacionada à assistência a saúde em adultos hospitalizados: uma revisão integrativa (Tese de Bacharelado, Universidade Federal do Rio Grande do Sul, Porto Alegre). https://lume.ufrgs.br/handle/10183/184413

Ercole, F. F., Chianca, T. C. M., Duarte, D., Starling, C. E. F., \& Carneiro, M. (2011). Infecção de sítio cirúrgico em pacientes submetidos a cirurgias ortopédicas: o índice de risco NNIS e predição de risco. Revista Latino-Americana de Enfermagem, 19(2), 269-276. https://doi.org/10.1590/S0104-11692011000200007

Ercole, F. F., Franco, L. M. C., Macieira, T. G. R., Wenceslau, L. C. C., de Resende, H. I. N., \& Chianca, T. C. M. (2011). Risk of surgical site infection in patients undergoing orthopedic surgery. Revista Latino-Americana de Enfermagem, 19(6), 1362-1368. https://doi.org/10.1590/s0104-11692011000600012

Ferraz, E. M., Bandeira Ferraz, A. A., Torres D’Albuquerque Coelho, H. S., Pereira Viana, V., Lopes Sobral, S. M., Marques Maia Vasconcelos, M. das D., \& Bacelar, T. S. (1995). Postdischarge surveillance for nosocomial wound infection: Does judicious monitoring find cases? American Journal of Infection Control, 23(5), 290-294. https://doi.org/10.1016/0196-6553(95)90059-4

Fontes, A. M. de S. (2010). Efetividade de intervenções na adequação da profilaxia antimicrobiana da infecção do sítio cirúrgico em um hospital universitário do Brasil (Tese de Mestrado, Universidade Federal de Uberlândia, Uberlândia). https://repositorio.ufu.br/handle/123456789/12690?locale=pt_BR 
Franco, L. M. de C., Ercole, F. F., \& Mattia, A. De. (2015). Infecção cirúrgica em pacientes submetidos a cirurgia ortopédica com implante. Revista Sobecc, 20(3), 163-170. https://doi.org/10.5327/z1414-4425201500030007

Gebrim, C. F. L., Rodrigues, J. G., Queiroz, M. N. R., Barreto, R. A. S. S., \& Palos, M. A. P. (2014). Antimicrobial prophylaxis analysis for prevention of surgical site infection in a Brazil centre-west hospital. Ciencia y Enfermeria, 20(2), 103-115. https://doi.org/10.4067/s0717-95532014000200011

Gonçalves, M. L. M., Silva, M. J. S. da, \& Torres, R. M. (2014). Antibioticoprofilaxia e tratamento de infecção do sítio cirúrgico na artroplastia de quadril. Revista Brasileira de Farmácia Hospitalar e Serviços de Saúde, 5(3), 18-22. http://rbfhss.saude.ws/revista/arquivos/2014050302000588BR.pdf

Greene, L. R. (2012). Guide to the elimination of orthopedic surgery surgical site infections: An executive summary of the Association for Professionals in Infection Control and Epidemiology elimination guide. American Journal of Infection Control, 40(4), 384-386. https://doi.org/10.1016/j.ajic.2011.05.011

Hochman, B., Nahas, F. X., Filho, R. S. de O., \& Ferreira, L. M. (2005). Desenhos de pesquisa. Acta Cirúrgica Brasileira, 20(2), 2-9. https://doi.org/10.1037/met0000082

Kirkland, K. B., Briggs, J. P., Trivette, S. L., Wilkinson, W. E., \& Sexton, D. J. (1999). The Impact of Surgical-Site Infections in the 1990s: Attributable Mortality, Excess Length of Hospitalization, And Extra Costs. Infection Control \& Hospital Epidemiology, 20(11), 725-730. https://doi.org/10.1086/501572

Le, J., Dong, Z., Liang, J., Zhang, K., Li, Y., Cheng, M., \& Zhao, Z. (2020). Surgical site infection following traumatic orthopaedic surgeries in geriatric patients: Incidence and prognostic risk factors. International Wound Journal, 17(1), 206-213. https://doi.org/10.1111/iwj.13258

Lima, Ana Lucia Lei Munhoz, \& de Oliveira, P. R. D. (2010). Update on Infections in Articular Prosthesis. Revista Brasileira de Ortopedia (English Edition), 45(6), 520-523. https://doi.org/10.1016/s2255-4971(15)30296-2

Lima, Ana Lúcia Lei Munhoz, Zumiotti, A. V., Uip, D. E., \& Silva, J. dos S. (2004). Fatores preditivos de infecção em pacientes com fraturas expostas nos membros inferiores. Acta Ortopédica Brasileira, 12(1), 32-39. https://doi.org/10.1590/s1413-78522004000100005

Maksimović, J., Marković-denić, L., \& Bumbaširević, M. (2008). Surgical Site Infections in Orthopedic Patients: Prospective Cohort Study. Croat Med J. 49, 58-65. https://doi.org/10.3325/cmj.2008.1.58

Mangram, A. J., Horan, T. C., Pearson, M. L., Silver, L. C., \& William, R. (1999). Guideline for Prevention of Surgical Site Infection, 1999. Centers for Disease Control and Prevention (CDC) Hospital Infection Control Practices Advisory Committee. AJIC, 27(2), 97-134. https://pubmed.ncbi.nlm.nih.gov/10196487/

Medina-Cuadros, M., Sillero-Arenas, M., Martínez-Gallego, G., \& Delgado-Rodríguez, M. (1996). Surgical wound infections diagnosed after discharge from hospital: Epidemiologic differences with in-hospital infections. American Journal of Infection Control, 24(6), 421-428. https://doi.org/10.1016/S01966553(96)90035-8

Mendes, E. C., Carvalho, M. de C., Carvalho, R. B., Ferraz, C. A., Souza, D. M. S. T., \& Schnaider, T. B. (2020). Técnicas de antissepsia em procedimentos cirúrgicos ortopédicos: Um estudo comparativo. Revista Brasileira de Ortopedia. 55(2), 56-62. https://doi.org/10.1055/s-0039-3400520

Miranda, A. R. de A. (2017). Aspectos Epidemiológicos Das Infecções De Sítio Cirúrgico Em Cirurgias Ortopédicas Com Implante Em Um Hospital De Reabilitação (Tese de Mestrado, Universidade Federal de Minas Gerais, Belo Horizonte). https://pesquisa.bvsalud.org/portal/resource/pt/biblio-983351

Moon, G. H., Cho, J.-W., Kim, B. S., Yeo, D. H., \& Oh, J.-K. (2019). Analysis of Risk Factors for Infection in Orthopedic Trauma Patients. J Trauma Inj, 32(1), 40-46. https://doi.org/10.20408/jti.2018.023

Nascimento, D. A. do, \& Corradi, J. G. (2019). Infecção de sítio cirúrgico em pacientes submetidos a cirurgias ortopédicas em um hospital estadual da região norte do Espírito Santo (Tese de Bacharelado, Faculdade Vale do Cricaré, São Mateus). https://repositorio.ivc.br/handle/123456789/328

Oliveira, A. C., \& Carvalho, D. V. (2004). Postdischarge surveillance: The impact on surgical site infection incidence in a Brazilian university hospital. American Journal of Infection Control, 32(6), 358-361. https://doi.org/10.1016/j.ajic.2004.02.009

Oliveira, P. R., Carvalho, V. C., Felix, C. da S., Paula, A. P. de, Santos-Silva, J., \& Lima, A. L. L. M. (2016). Infecção de sítio cirúrgico após fixação de fraturas fechadas e expostas - Incidência e perfil microbiológico. Revista Brasileira de Ortopedia, 51(4), 396-399. https://doi.org/10.1016/j.rbo.2015.09.010

Onyekwelu, I., Yakkanti, R., Protzer, L., Pinkston, C. M., Tucker, C., \& Seligson, D. (2017). Surgical Wound Classification and Surgical Site Infections in the Orthopaedic Patient. Journal of the American Academy of Orthopaedic Surgeons, 1(3), 1-10. https://doi.org/10.5435/jaaosglobal-d-17-00022

Ortega, G., Rhee, D. S., Papandria, D. J., Yang, J., Ibrahim, A. M., Shore, A. D., Makary, M. A., \& Abdullah, F. (2012). An evaluation of surgical site infections by wound classification system using the ACS-NSQIP. Journal of Surgical Research, 174(1), 33-38. https://doi.org/10.1016/j.jss.2011.05.056

Ren, M., Liang, W., Wu, Z., Zhao, H., \& Wang, J. (2019). Risk factors of surgical site infection in geriatric orthopedic surgery: A retrospective multicenter cohort study. Geriatrics and Gerontology International, 19(3), 213-217. https://doi.org/10.1111/ggi.13590

Ribeiro, J. C., Dos Santos, C. B., Bellusse, G. C., Da Fonseca Rezende, V., \& Galvão, C. M. (2013). Occurrence and risk factors for surgical site infection in orthopedic surgery. ACTA Paulista de Enfermagem, 26(4), 353-359. https://doi.org/10.1590/s0103-21002013000400009

Roth, J. A., Juchler, F., Dangel, M., Eckstein, F. S., Battegay, M., \& Widmer, A. F. (2019). Frequent door openings during cardiac surgery are associated with increased risk for surgical site infection: A prospective observational study. Clinical Infectious Diseases, 69(2), 290-294. https://doi.org/10.1093/cid/ciy879

Vieira, D. A. R., Cristianismo, R. S., Campos, F. F., Costa, M. C. P. da, \& Lucas, T. C. (2021). Infecção de sítio cirúrgico em osteossíntese de fêmur: incidência e fatores associados. Cogit. Enferm. 26(76087). https://doi.org/10.5380/ce.v26i0.76087 